\title{
Object Dependent Properties of Mirrors for PV Applications Studied Under Accelerated Weathering Protocols
}

\author{
Ian V. Kidd, Laura S. Bruckman, Myles P. Murray and Roger H. French \\ Case Western Reserve University, Cleveland, OH, 44106, USA
}

\begin{abstract}
Lifetime and degradation science (L\&DS) has been implemented over the past two years to explore exposure protocols for accelerated weathering of back-surface aluminum mirrors for photovoltaic applications and to apprise PV developers of their implications. Using weathering protocols that induce degradation through heat, humidity, ultraviolet light and exposure to corrosive environments, a variety of degradation modes have been observed. In order to characterize changes in the object-dependent optical properties of these mirrors, a careful accounting of reflected, absorbed and scattered light was performed. Metrics for comparing the degradation observed in various sets of samples with different protective coatings were developed. These metrics can be used to track, correlate and predict performance of systems exposed in real-world conditions. Using a stress and response framework, observations of sample performance were made at multiple steps of exposure in order to monitor the progression of damage accumulation. This allows for correlation to outdoor exposure results and provides a pathway for system lifetime performance prediction. Acrylic back surface mirrors exposed to accelerated weathering protocols showed an increase in scatter, interfacial absorptance and a reduction in specularity. This L\&DS approach to technology development allows for confidence when entering new markets and mitigates risks associated with premature failure due to improper material substitution.
\end{abstract}

Index Terms-Potovoltaic effects, mirrors, degradation, lifetime estimation, energy.

\section{INTRODUCTION}

Object-dependent properties are optical properties, such as reflectance, absorptance, transmission and scatter, which are dependent on the size, shape and roughness of each material. This is similar to extrinsic variables where the exhibited properties depend on the size and shape. Aluminum back sheets, for example, show object-dependent properties when too thin of a layer is deposited, causing transmission through the mirror. Photovoltaic (PV) back surface mirrors can consist of a tri-layered system of an acrylic front surface, an aluminum back surface, and a protective coating. Within each of these layers, different degradation mechanisms and rates can be observed in different weathering protocols. Heat, humidity, ultraviolet light (UV) and corrosive environments can induce different degradation pathways and rates in each material. These pathways can be evaluated at different stages using a stress and response $(\mathrm{R}(\mathrm{S}))$ framework [1]. The use of object dependent properties enables the abilit to isolate the impact of individual variables and determines the impact on the overall reflectance of the mirror. In considering objectdependent properties, the mirror can be thought of as having three regions: an acrylic substrate, an aluminum back surface mirror and a protective coating.

Polymers, including those in the acrylic substrate, are highly susceptible to degradation from exposure to UV light. The degradation arises from the high energy of the UV light present in the solar spectrum which can damage or break the carboncarbon bonds in polymer [2], [3]. This mode of degradation can be limited by the addition of a hydroxydephenyl benzotriazole based UV stabilizer, which is sacrificially degraded by the UV light in order to protect the polymer matrix [4]. In acrylic, this degradation is observable as yellowing and haze.

Degradation of the aluminum back surface reflector can also limit the reflectance of the mirror. In aluminum mirrors the two main concerns for degradation are surface roughness and corrosion. Surface roughening can lead to geometric absorptance-self-shadowing or scatter if RMS roughness is large compared to wavelength. Absorptance is the ratio of the radiation absorbed to radiation of the incident beam. Metallization roughening in mirrors has been studied in the presence of corrosive environments and under vacuum UV and UV irradiation. The effects of surface contaminants have also been studied under these conditions [5], [8]. If oxidization or corrosion occurs, optical losses from increased scattering or increased absorbption from the oxide could result.

Protective coatings, consisting of $\mathrm{TiO}_{2}$ and $\mathrm{ZnO}$ in a polymer binder provide opacity and slow yellowing, mildew and UV degradation to the underside of the mirror. These coatings have been shown to last 25 years or more before failure of the coating occurs and corrosion of the aluminum surface occurs [9].

The aforementioned degradation mechanisms have been induced using multi-step exposure protocols consisting of varying environments of heat, humidity, UV light and corrosive salt that result in accelerated weathering. The resulting loss of specularity and increased scatter and absorptance can be characterized using object-dependent properties. By determining the object-dependent properties the performance of the mirror over its entire lifetime can be predicted.

\section{Methodology}

Three separate accelerated weathering protocols, Hot QUV, ASTM G154 cycle 4 with QUV and Q-fog salt-fog could be used. Hot QUV (ASTM G154 without condensing humidity) was performed using UVA340 fluorescent lamps that match the high energy UV range of the solar spectrum with conditions of $1.55 \mathrm{w} / \mathrm{m}^{2} / \mathrm{nm}$ at $340 \mathrm{~nm}$ and a chamber temperature of $70^{\circ} \mathrm{C}$. 
ASTM G154 cycle 4 with QUV was conducted in accordance with ASTM G154 Cycle 4 with condensing humidity. Maximum irradiance, at $340 \mathrm{~nm}$, was set at $1.55 \mathrm{w} / \mathrm{m}^{2} / \mathrm{nm}$ and chamber temperature was set at $70^{\circ} \mathrm{C}$, condensing humidity was obtained at $50^{\circ} \mathrm{C}$. Salt-fog corrosion exposures were performed in a Q-Labs Q-FOG cyclic corrosion cabinet. The salt-fog solution was $5 \% \mathrm{NaCl}$ and the cabinet temperature was $35^{\circ} \mathrm{C}$ as prescribed in ASTM B117. Salt-fog corrosion according to ASTM B117 does not correlate to any real-world exposure other than submersion in tidal water, but as a highly accelerated stress test may be useful for screening candidate reflector compositions. However, this is a widely accepted industrial engineering control and allows comparability. Mirror yellowing is quantified using the mirror yellowing index $\left(M_{y} i\right)$ which uses the reflectance value at three different wavelengths as seen in Equation 1

$$
R_{y i}=\frac{R_{630}-R_{420}}{R_{500}}
$$

The data was then analyzed using measurably properties of reflectance $(R)$, absorptance $(A)$, transmission $(T)$ and scattering $(S)$ account for all the light in the system (Equation 2).

$$
R+A+T+S=1
$$

In the case of mirrors, however, transmission is equal to 0 since the aluminum surface is thick enough to prevent transmission. Reflectance measurements were taken using an Aglient CARY 6000i spectrophotometer with a diffuse reflectance accessory (DRA1800) so both total hemispherical and diffuse reflectance could be measured. Scattering was measured using a Scattermaster ScatterScope 3D with the data being fit to the bidirectional scatter distribution function (BSDF), which is given by the Harvey-Shack approximation.

$$
B S D F=\frac{A}{B+\beta+\beta_{0}^{g}}[10]
$$

In Equation 3 , the $\beta$ vector represents the projection of the unit vector of the scattering direction onto the tangent plane and the $\beta_{0}$ vector represents the projection of the unit vector in the specular direction onto the tangent plane. $A, B$ and $g$ are fitting parameters that allow the model to accurately portray the scattering data. $A$ determines the height of the fitting curve, $B$ determines the inflection point where the curve transitions from flat to sloped and $\mathrm{g}$ determines the slope of the roll-off. Absorptance is then calculated using Equation 2. The BSDF is then imported into TracePro, a ray tracing CAD program, where it can be applied as a surface property to the mirrors. By using a ray trace representative of the suns spectra, a visual and numerical approximation can be made to determine the efficiency and performance of different materials. It is important to note that in this simulation, a perfect absorber was used to represent the solar cell to isolate the effect of the mirrors.

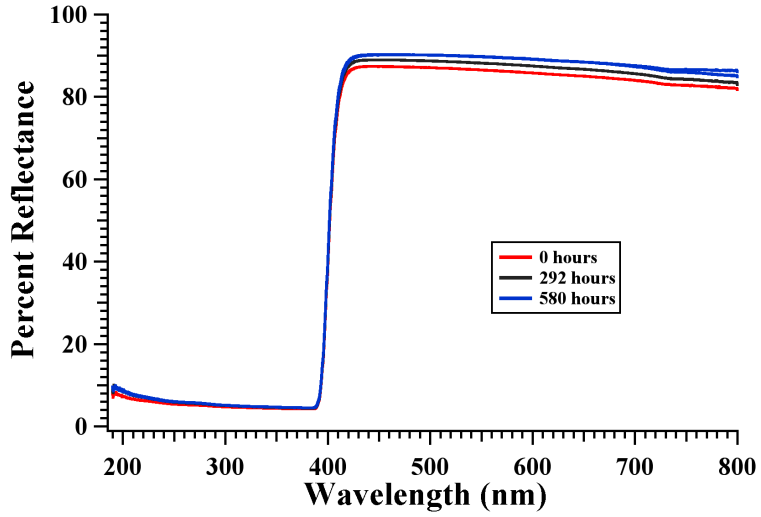

Fig. 1. Reflectance of UVA, an acrylic with a UV stabilizing package, after exposure to Hot QUV over the wavelengths of $200-800 \mathrm{~nm}$

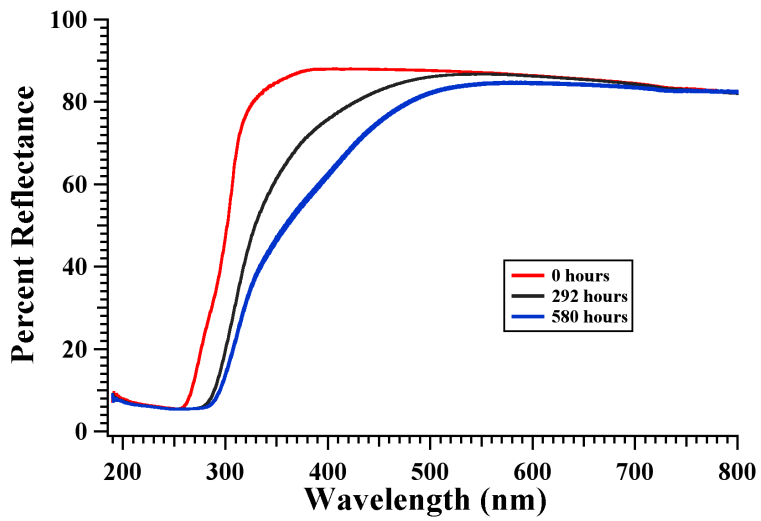

Fig. 2. Reflectance of UVR (no stabilizer package) after exposure to Hot QUV over the wavelengths of $200-800 \mathrm{~nm}$

\section{RESULTS}

Reflectance and scattering measurements were used to analyze each exposure protocol. This is done to isolate different variables to get a better understanding of the correlation of object-dependent properties. Figure 1 shows the results of UVA mirrors exposed to Hot QUV for the wavelengths of 200$800 \mathrm{~nm}$. This contrasts with 2 which shows the degradation of UVR mirrors exposed to Hot QUV for wavelengths of 200-800 [1]. This propmted a side by side exposure to both examine the differences between UVA and UVR mirrors as well as provide a amply degraded sample in the form of the UVR mirror to model our system.

The addition of humidity in the form of ASTM G154 cycle 4 further changes the rate of reflectance loss, as seen in Figure 3

Figure 3 is important as it shows that the use of ASTM G154 cycle 4 provides the fastest degradation rates while still maintaining the same degradation mechanisms, which is seen by the same curvature of the reflectance curve.

From this point on, all analysis is focused on comparing unexposed samples to samples with 868 hours of exposure as that is where the diffuse only reflectance exceeds the error 


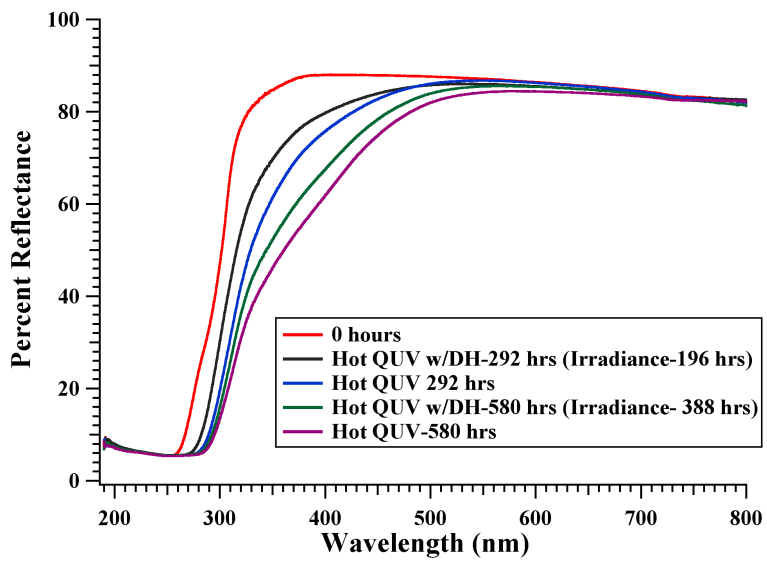

Fig. 3. The addition of humidity from ASTM G154 increases the rate of degradation per unit time of irradiance exposure over the $200-800 \mathrm{~nm}$ wavelengths

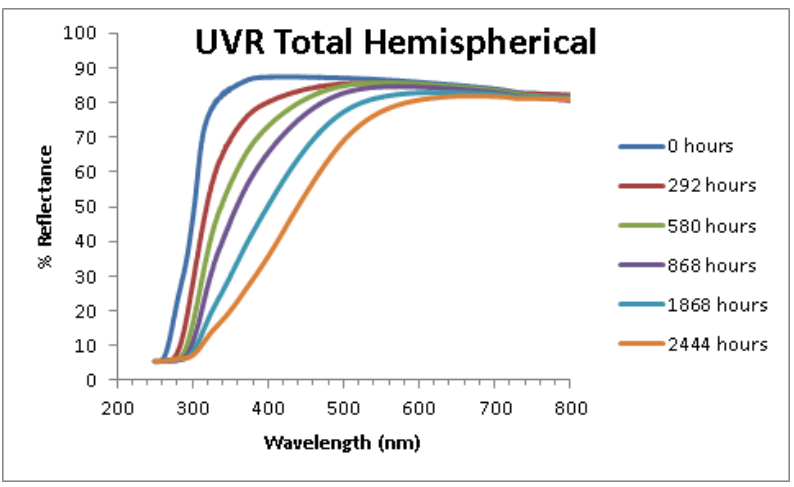

Fig. 4. Total hemispherical reflectance from $200-800 \mathrm{~nm}$ as exposure dose increase, the samples have $0,292,580,868,1868$ and 2444 hours of exposure to ASTM G154 cycle 4, respectively.

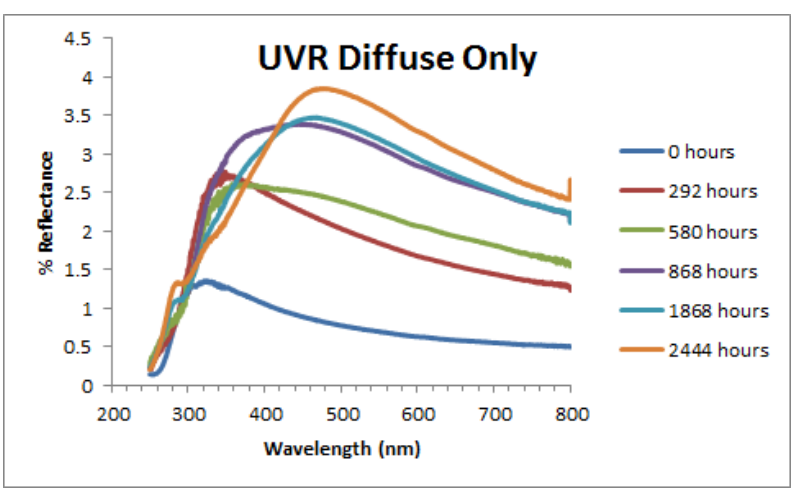

Fig. 5. Diffuse only reflectance from $200-800 \mathrm{~nm}$ as exposure dose increase, the samples have $0,292,580,868,1868$ and 2444 hours of exposure to ASTM G154 cycle 4 respectively.

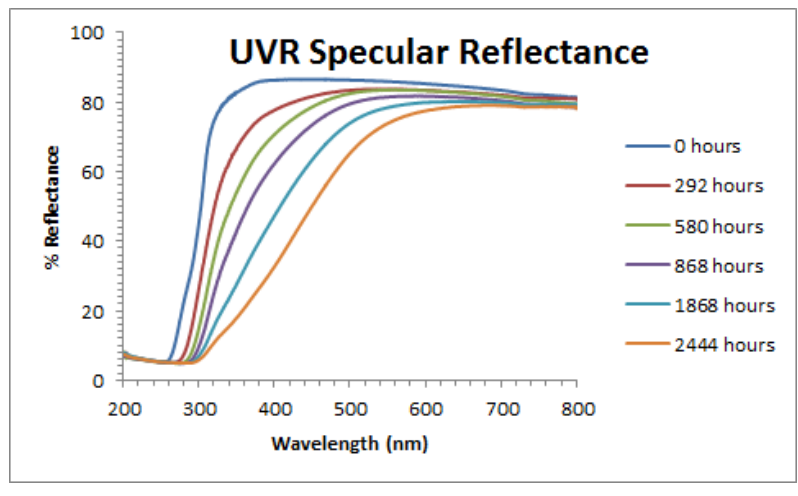

Fig. 6. UVR specular reflectance from 200- $800 \mathrm{~nm}$ calculated from 4 and 5 (total hemispherical minus diffuse only).

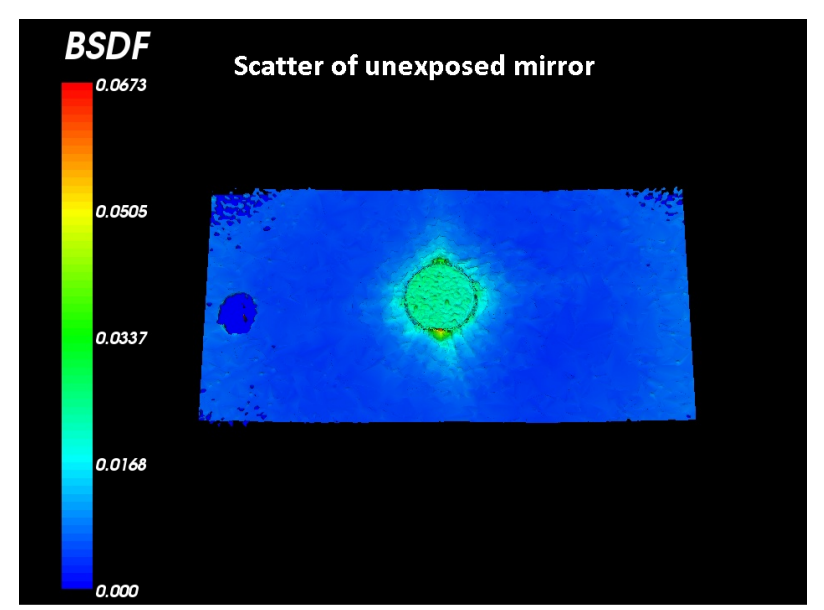

Fig. 7. Scatter of sample with no exposure. Note both small radius of scatter and the low intensity of scatter, indicating a mirror with both high overall reflectance and good specularity.

in the equipment and becomes statistically signigicant. The becomes apparent in Figure 5 where the diffuse reflectance exceeds $2 \%$. The specular reflectance curves seen in Figure 6 comes from measuring the total hemispherical reflectance in Figure 4 and subtracting the diffuse reflectance from Figure 5

With the ability to measure scatter, which is represented visually in Figure 7 and figure 8 and specular reflectance in Figure 6, absorptance can be calculated, which is done on the ScatterMaster and fitting to the BSDF is performed as seen in Table [ using an inverse power law with $\beta$ and $\beta_{0}$ coming from the Harvey-Shack approximation.

TABLE I

BSDF OF UVR AS A FUNCTION OF EXPOSURE TIME

\begin{tabular}{|c|c|c|}
\hline Exposure & Mean g-Value & Standard Deviation \\
\hline 0 & 2.585 & 0.29 \\
292 & 2.4284 & 0.16 \\
580 & 2.2256 & 0.23 \\
868 & 2.1543 & 0.18 \\
1868 & 2.0815 & 0.11 \\
\hline & \multicolumn{2}{|l}{}
\end{tabular}

The scattering ray trace between the unexposed and exposed 


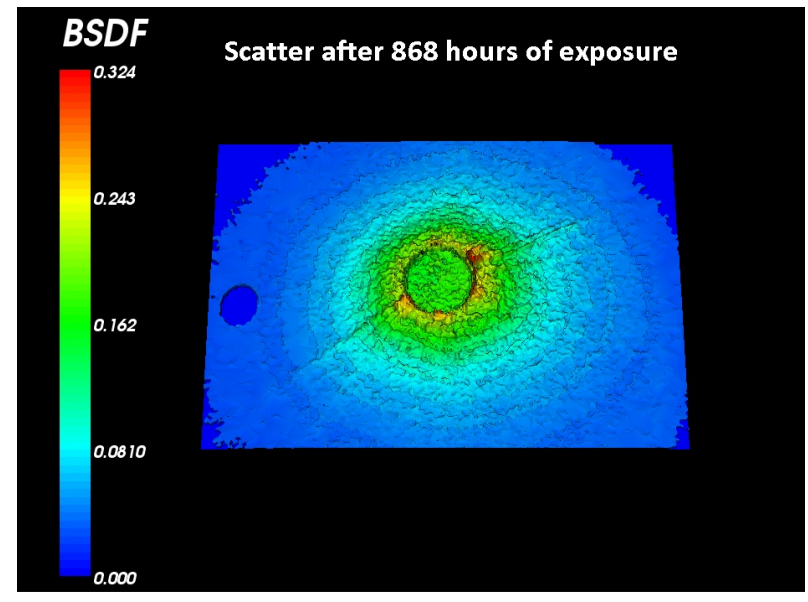

Fig. 8. BSDF of UVR mirror exposed to 868 hours of blank showing that the scatter radius has increased, which indicates that light is being scattered at an angle farther away from specular. It is important to note that scale on the left, corresponding to the amplitude of the BSDF has increased compared to that of 7 to account for the increased scattering intensity.

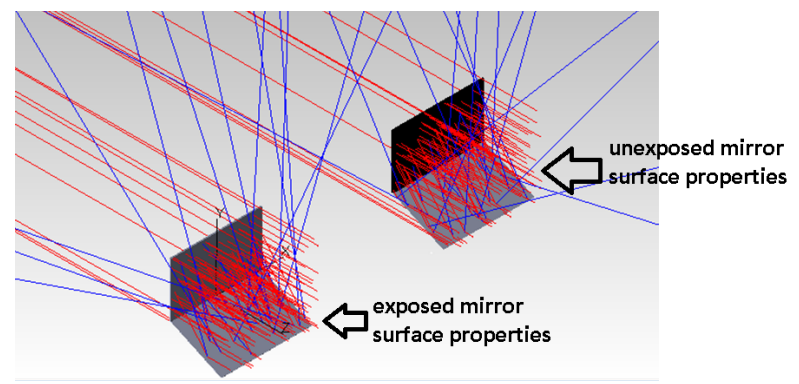

Fig. 9. TracePro representation of mirror surface properties applied. Rays are a simulation of the sun and have variable energies that match the solar spectru. note that blue lines are scattered rays being deemed "scattered" due to their energy before and after interaction with the mirror.

samples in Figure 9 look very similar but from the spectra taken it is known that losses must come from somewhere. These losses were attributed to an increased absorbtance of the mirrors and confirmed by Figure 10 and Figure 11

To see the impact of the mirror properties, the irradiance of the perfect absorber was also analyzed. The perfect absorber confirms the result from the mirrors and indicates a $1-2 \%$ loss in overall power potential. The trace of the perfect absober is shown in Figure 12

\section{DISCUSSION}

The high rate of degradation seen in samples exposed to ASTM G154 cycle 4 and Hot QUV indicate that the main contributor to the loss of reflectance of the mirrors is the yellowing of the acrylic. This is emphasizes the importance of the UV stabilizing package in the acrylic. Yellowness index, calculated using Equation 11 can provide insights into the time and rate of change of degradation modes in exposed samples. In samples without the stabilizing package (UVR), the critical exposure dose was determined to be $1000 \mathrm{GJ} / \mathrm{m}^{2}$, which is equivalent to 8-12 years of tracked exposure in Arizona. The

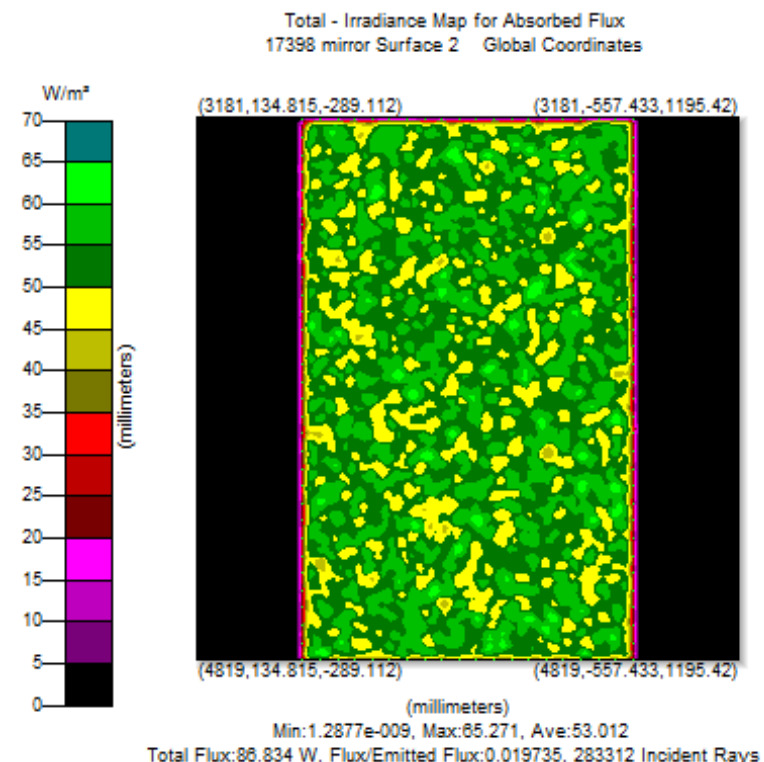

Fig. 10. Absorbance of unexposed sample with a average absorbance of 53 $\mathrm{w} / \mathrm{m}^{2}$.

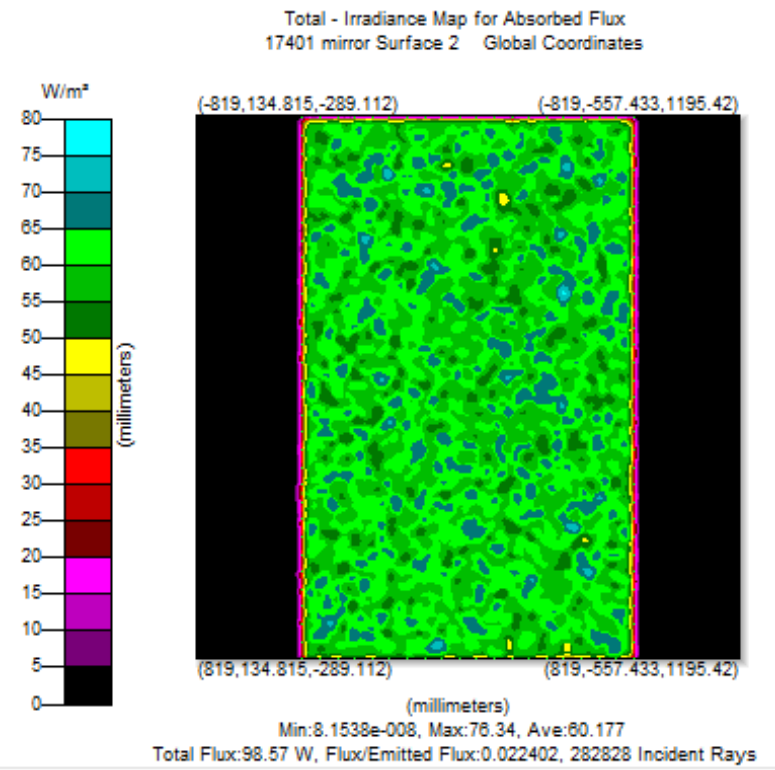

Fig. 11. Absorbance of sample exposed to 868 hours, with a average absorbance of $60 \mathrm{w} / \mathrm{m}^{2}$ corresponding to $12 \%$ more than the unexposed sample in Figure 10

UVA, which has the stabilizer package does not show this change until $2270 \mathrm{GJ} / \mathrm{m}^{2}$, which is a lifetime of over 20 years tracked performance in Arizona. The absorption of the UV light by the stabilizer package also protects the actual panel, which can decrease in performance from UV exposure.

For analysis of object dependent properties, the UVR was chosen to obtain quicker and more drastic changes. Even using the less stable acrylic, surface roughening has been observed to be primarily a function of the acrylic substrate rather than the aluminum back sheet. This has been deduced by the degrada- 


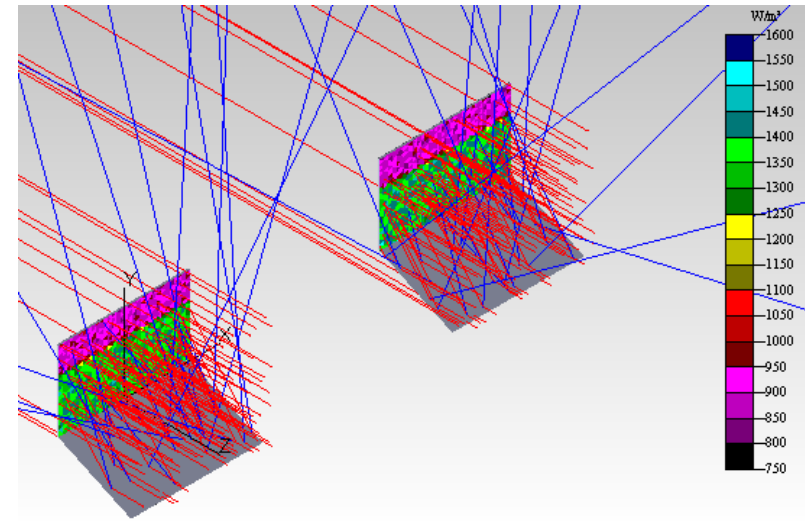

Fig. 12. Absorbance of the perfect absorber. Though hard to see, the system with the exposed mirror (sa17401) has lower absorptance in the simulated cell, meaning less power output.

tion seen when comparing UVR to UVA acrylic. Confirmation of this hypothesis will come as isolated acrylic is exposed. The BSDF images in Figure 7 and Figure 8 shows a clear increase in both the amplitude and radius of scattering. This indicates that while the acrylic may be functional, processing techniques that minimize sample roughness are important to both the initial and lifetime performance of mirrors. Most importantly, the amount of scattering can be quantified, allowing the formation of object-dependent properties. By modeling the unexposed and exposed mirrors in TracePro, the ray traces in figure 9 initially look remarkably similar in the number of scattered rays. However, TracePro sorts the waves based off of energy so while it is true that the scattered rays have lower energy, it is required to break an arbitrary threshold before it is considered scattered. Figure 10 and Figure 11 makes this apparent as the absorptance of the exposed mirror is absorbs approximately $12 \%$ more energy than the unexposed mirror. When this loss is transferred to the cell, the overall energy loss of the cell is approximately $2 \%$, which is calculated from the absorbed incidence of the absorber in Figure 12

Reflectance and BSDF analysis can allow for quantitative prediction of system performance over product lifetime because object dependent properties allow for quantitative optical modeling of systems through time. This gives developers greater confidence in predicting degradation and lifetime performance prior to entering the PV market. In addition, object dependent properties break the PV system into individual components allowing manufacturers to make small changes without retesting of the entire module. By isolating the components, performance in individual sections can be monitored and the cost of running exposures to test the lifetime performance of changing a single component can be greatly reduced. This reduction in cost can spur innovation and faster change in preparing PV modules to meet lifetime predictions and warranties.

\section{ACKNOWLEDGMENT}

We would like to thank the SDLE center and all of the members for helping both directly and indirectly on this research. This research was made possible by Ohio Third Frontier under the grants of Ohio Third Frontier Tech 11-060 and Ohio Third Frontier Tech 12-004

\section{REFERENCES}

[1] M. P. Murray, L. S. Bruckman, R. H. French, "Photodegradation in a stress and response framework: poly(methyl methacrylate) for Solar Mirrors and Lens," J. Photonics for Energy, 2(1), 022004 (Nov 19, 2012).

[2] R. H. French, J. M. Rodrguez-Parada, M. K. Yang, R. A. Derryberry, N. T. Pfeiffenberger, "Optical properties of polymeric materials For concentrator photovoltaic systems," Sol. Energy Mater. Sol. Cells, 95, 2077-86, (2011),

[3] M. Pecht, "Product reliability, maintainability, and supportability handbook," 2009.

[4] J. E. Moore, J. E. Pickett, "Photodegradation of uv screeners, polymer degradation and stability," Polymer Degradation and Stability, 42(3):231244, 1993.

[5] R.W. Dreyfus, R.J. Von Gutfeld, and S.C. Wallace, "Aluminum mirror degradation in a vuv laser," Optics Communications, 9(4):342345,1973.

[6] T.E. Madey, N.S. Faradzhev, B.V. Yakshinskiy, and NV Edwards, "Surface phenomena related to mirror degradation in extreme ultraviolet (euv) lithography," Applied Surface Science, 253(4):16911708,2006.

[7] R.P. Young, "Low-scatter mirror degradation by particle contamination," Optical Engineering, 15(6):156516156516, 1976.

[8] R.P. Young, "Mirror scatter degradation by particulate contamination, In Society of Photo-Optical Instrumentation Engineers (SPIE) Conference Series, volume 1329, 246254, 1990.

[9] K.D.Weiss, "Paint and coatings: A mature industry in transition," Progress in Polymer Science, 22(2):203245, 1997.

[10] J.E. Havey, R.V. Shack, "Aberrations of diffraced wave fields," Applied Optics, Vol. 17, Issue 18, pp. 3003-3009 (1978) 